HNO 2021 · 69:693-694

https://doi.org/10.1007/s00106-021-01080-7

Angenommen: 10. Mai 2021

(c) Springer Medizin Verlag $\mathrm{GmbH}$, ein Teil von Springer Nature 2021

\section{Neue Entwicklungen in Phonochirurgie und Laryngologie}

\author{
Christoph Arens ${ }^{1}$. Markus Gugatschka ${ }^{2}$ \\ ' Universitätsklinik für Hals-, Nasen- und Ohrenklinik, Kopf- und Halschirurgie, Universitätsklinikum \\ Magdeburg, Otto-von-Guericke-Universität Magdeburg, Magdeburg, Deutschland \\ ${ }^{2}$ Klinische Abteilung für Phoniatrie, Hals-Nasen-Ohren-Universitätsklinik, Medizinische Universität Graz, \\ Graz, Österreich
}

Die Stimme ist unser wichtigstes Kommunikationsmittel. Diese Tatsache hat in modernen Dienstleistungsgesellschaften nochmals eine Aufwertung erfahren. Die Stimme transportiert aber nicht nur linguistische Informationen, sie verrät auch viel über den jeweiligen Menschen, über sein Geschlecht, die Stimmung oder die Intention, wie etwas gesagt wird.

\section{》) Stimmliche Einschränkungen haben auch erhebliche ökonomische Folgen}

Stimmliche Einschränkungen haben aber auch erhebliche ökonomische Folgen, wenngleich konkrete Zahlen nur für die USA vorliegen. Eine viel zitierte Studie von Ruben schätzt, dass Kommunikationsstörungen bis zu 2,5\% des Bruttoinlandsprodukts (BIP) entsprechen [1]. Epidemiologische Zahlen hierzu variieren stark und hängen eng mit der Fragstellung zusammen. Befragt nach der Selbsteinschätzung einer Stimmstörung gaben 7,6\% der Erwachsenen in den USA ein Problem innerhalb eines Jahres an (National Health Interview Survey) [2]. In einer europäischen Studie (Stockholm Public Health Cohort 2010), fand sich eine Prävalenz von knapp 17\% [3]. Eine sehr gut untersuchte Gruppe in Zusammenhang mit beruflichem Stimmgebrauch stellt die der Lehrer*innen dar. Eine große Studie in den USA zeigte, dass diese eine Lebenszeitprävalenz an Stimmstörungen von $58 \%$ aufwies (im Vergleich zu $28 \%$ in einer Kontrollgruppe) [4].

In jedem Fall liegen Stimmstörungen aber Veränderungen in Morphologie und
Funktion des Kehlkopfs oder an der Stimmbildung beteiligter Strukturen zugrunde. Technische Innovationen und Weiterentwicklungen der letzten Jahre eröffneten faszinierende neue Einblicke und ermöglichten neue Möglichkeiten zur Diagnostik und Behandlung bekannter Krankheitsbilder.

Das vorliegende Schwerpunktheft soll diese Entwicklungen widerspiegeln. Der Bogen spannt sich dabei von der Stimmlippenphysiologie über Standards in der Stimmdiagnostik und Phonochirurgie hin zu technischen Innovationen wie beispielsweise Mikroelektronik in der Neurolaryngologie. Der erste Artikel (Gugatschka et al.) beschäftigt sich mit den zellulären Grundlagen der Stimmlippen und stellt Invitro-Modelle vor, mit welchen Stimmlippenerkrankungen erstmalig molekular untersucht werden können. Ein besseres Verständnis der zellulären Abläufe soll künftig eine biologisch untermauerte Grundlage für die Etablierung von neuen Modellen darstellen.

Eine umfassende Stimmdiagnostik ist elementarer Bestandteil jeglicher Therapie und sollte routinemäßig zu einem perioperativen Prozedere gehören. $B$. SchneiderStickler stellt entsprechende multidimensionale Protokolle vor, deren Standardisierung ein wichtiger Schritt in puncto Qualitätssicherung ist.

Schöninger et al. befassen sich mit dem innovativen Bereich der Kontaktendoskopie mit Narrow Band Imaging (NBI) als diagnostischem Verfahren. Dies ist eine Technik, die während der Mikrolaryngoskopie eingesetzt wird, um schon vor der Operati- on eine Entscheidung über den Charakter der Läsion treffen zu können.

\section{») In den letzten Jahren wurde ein Trend zu ambulanten und tagesklinischen Behandlungen augenscheinlich}

In den letzten Jahren wurde ein Trend zu immer kürzeren Krankenhausaufenthalten bzw. ambulanten und tagesklinischen Behandlungen augenscheinlich. „Officebased procedures" setzen sich dank hochauflösender Endoskope auch in unseren Kliniken immer mehr durch. J. Bohlender gibt einen umfassenden Überblick über Indikationen, Limitationen, Techniken und das notwendige Equipment.

Für den Bereich der Laryngeal-Framework-Chirurgie geben $M$. Hess et al. einen nahezu vollständigen Überblick über Indikationen, Techniken und Limitationen inkl. perioperativem Management von Thyroplastiken.

Ein besonders spannendes Gebiet, welches sich in den letzten Jahren extrem schnell entwickelt hat, ist das der Neurolaryngologie. Der Beitrag von A. Müller zeigt auf, wie neueste Entwicklungen der Mikroelektronik ein neues Spektrum an Behandlungsoptionen erschlossen haben. Damit einher ging auch ein verstärkter Einsatz der dementsprechenden Diagnostik, Methoden wie die Larynxelektromyographie (LEMG) wurden zuletzt angewendet wie nie zuvor.

Jede Auswahl stellt auch immer eine Selektion und daher einen Ausschnitt dar. Wir hoffen, dass es uns trotzdem gelungen ist, unsere Begeisterung für dieses faszi- 


\section{Einführung zum Thema}

nierende Teilgebiet mit den Leser*innen zu teilen.

\section{Korrespondenzadresse}

Prof. Dr. med. Christoph Arens

Universitätsklinik für Hals-, Nasen- und Ohrenklinik, Kopf- und Halschirurgie, Universitätsklinikum Magdeburg, Otto-vonGuericke-Universität Magdeburg Leipziger Str. 44, 39120 Magdeburg, Deutschland christoph.arens@med.ovgu.de

Univ. Prof. PD Dr. med. univ. et scient. med. Markus Gugatschka

Klinische Abteilung für Phoniatrie, HalsNasen-Ohren-Universitätsklinik, Medizinische Universität Graz

Auenbruggerplatz 26, 8036 Graz, Österreich markus.gugatschka@medunigraz.at

Interessenkonflikt. C. Arens und M. Gugatschka geben an, dass kein Interessenkonflikt besteht.

\section{Literatur}

1. Ruben RJ (2000) Redefining the survival of the fittest: communication disorders in the 21st century. Laryngoscope 110(2 Pt 1):241-245

2. Bhattacharyya N (2014) The prevalence of voice problems among adults in the United States. Laryngoscope 124(10):2359-2362

3. Lyberg-Ahlander V, Rydell R, Fredlund P, Magnusson C, Wilen S (2018) Prevalence of voice disorders in the general population, based on the Stockholm publichealth cohort. J Voice 33(6):900-905

4. Roy N, Merrill RM, Thibeault S, Parsa RA, Gray SD, Smith EM (2004) Prevalence of voice disorders in teachers and the general population. J Speech Lang Hear Res 47(2):281-293

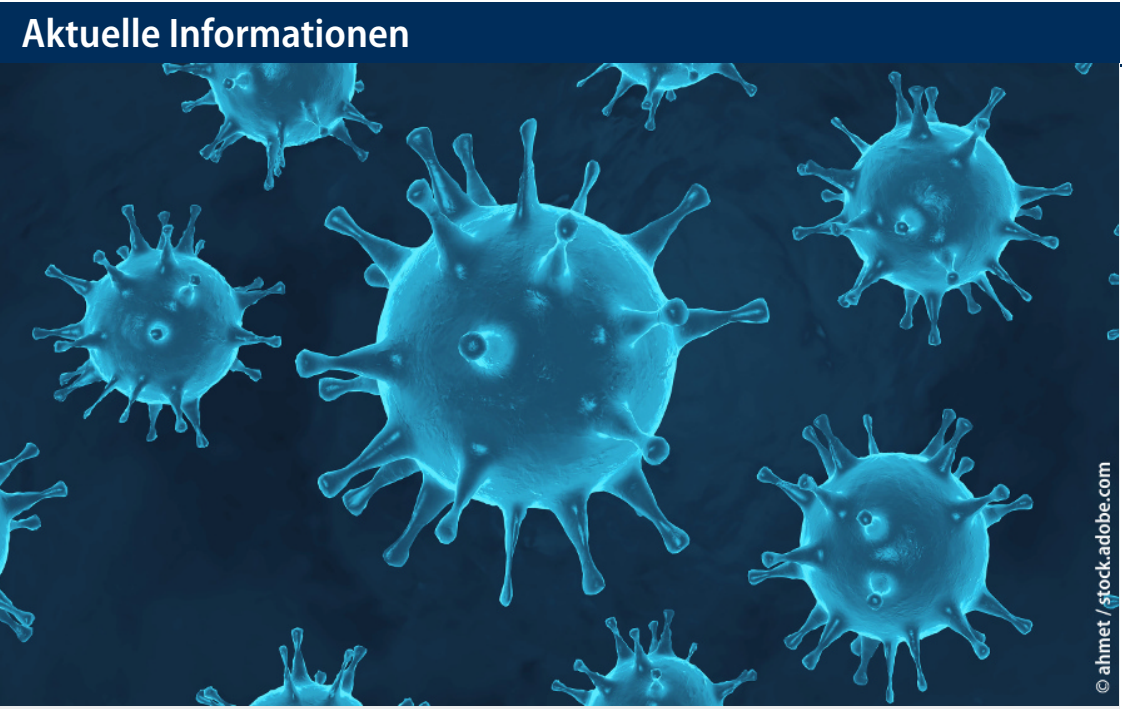

\section{Aktuelle Beiträge zu COVID-19 in der HNO}

Springer Nature und Springer Medizin unterstützen die globale Reaktion auf die COVID-19-Pandemie, indem ein schneller und direkter Zugang zu den neuesten verfügbaren Forschungsergebnissen und Daten ermöglicht wird.

Auf der Homepage SpringerMedizin.de finden Sie ein immer aktuelles Dossier mit Beiträgen, Forschungsarbeiten und Ergebnissen zu SARS-CoV-2 sowie relevanten Links. Darin z.B. auch die folgenden, frei zugänglichen Beiträge aus der HNO zu COVID-19.

- Budenosid bei COVID-19, https://doi.org/10.1007/s00106-021-01069-2

- Das erste Jahr der Pandemie mit SARS-CoV-2 - Auswirkungen auf die Hals-Nasen-OhrenHeilkunde, https://doi.org/10.1007/s00106-021-01015-2

- Zukunftsweisende Therapieansätze bei Riechstörungen: Elektrische Stimulation, Stammzelltherapie und Transplantation von Riechepithel - eine Übersicht, https://doi.org/10.1007/s00106-021-01060-x

- Auswirkungen der SARS-CoV-2-Pandemie auf die universitäre Hals-Nasen-Ohren-Heilkunde im Bereich der Forschung, Lehre und Weiterbildung, https://doi.org/10.1007/s00106-02101001-8

- Elektronisches Lernen für Studenten in der Hals-Nasen-Ohren-Heilkunde durch Nutzung des Content-Management-Systems ILIAS, https://doi.org/10.1007/s00106-021-01008-1

- Tröpfchenexposition bei Tracheotomie, https://doi.org/10.1007/s00106-021-01040-1

- Ertaubung nach COVID-19?, https://doi.org/10.1007/s00106-021-01040-1

Diese Beiträge werden wir in der August-Ausgabe der HNO abdrucken.

Das Dossier zu Coronavirus / Covid-19 von Springer Medizin finden Sie hier: www.springermedizin.de/covid-19 